\title{
Post-annealed Aluminum-Doped Zinc Oxide/Tin-Doped Indium Oxide Bilayer Films for Low Emissivity Glass
}

\author{
Shang-Chou Chang ${ }^{12, *}$ and Huang-Tian Chan ${ }^{2}$ \\ ${ }^{1}$ Department of Electrical Engineering, Kun Shan University, Tainan City 71010, Taiwan \\ ${ }^{2}$ Green Energy Technology Research Center, Kun Shan University, Tainan City 71010, Taiwan \\ *E-mail: jchang@mail.ksu.edu.tw
}

doi: $10.20964 / 2020.05 .75$

Received: 5 January 2020 / Accepted: 4 March 2020 / Published: 10 April 2020

\begin{abstract}
This study uses post-annealed aluminum-doped zinc oxide /tin-doped indium oxide bilayer (AZO/ITO) films for low emissivity glass. The AZO/ITO films are deposited on glass substrates using in-line sputtering. The AZO/ITO films are subjected to either vacuum or hydrogen plasma annealing. The microstructure, the visible transmittance, the electrical properties and the emissivity of as-deposited, vacuum-annealed and plasma-annealed AZO/ITO films are measured. Hydrogen plasma annealing changes the surface morphology of AZO/ITO films. The average visible transmittance increases and the electrical resistivity and emissivity decrease for AZO/ITO films after vacuum or plasma annealing. The vertical cross-section and surface morphology for vacuum-annealed AZO/ITO films is similar to that for as-deposited films. The surface roughness of AZO/ITO films increases and polygonal grains form after plasma annealing. The electrical resistivity of the vacuum-annealed and hydrogen plasmaannealed AZO/ITO films is 59\% and 63\% less than the value for as-deposited films. The average visible transmittance of the vacuum-annealed and hydrogen plasma-annealed AZO/ITO films is $8.64 \%$ and $12.71 \%$ greater than the value for as-deposited films. The emissivity of vacuum-annealed and hydrogen plasma-annealed samples is 2.3 times and 2.56 times less than that of as-deposited AZO/ITO films. The emissivity and the average visible transmittance for vacuum-annealed and hydrogen plasma-annealed AZO/ITO films is 0.09 and $81.58 \%$ respectively, which is within the range for commercial low emissivity materials.
\end{abstract}

Keywords: AZO/ITO; vacuum annealing; hydrogen plasma annealing; low emissivity glass

\section{$\underline{\text { FULL TEXT }}$}

(C) 2020 The Authors. Published by ESG (www.electrochemsci.org). This article is an open access article distributed under the terms and conditions of the Creative Commons Attribution license (http://creativecommons.org/licenses/by/4.0/). 